\title{
A Mereology for Connected Structures
}

\author{
Michael GRÜNINGER ${ }^{\text {a }}$, Carmen CHUI ${ }^{\text {a }}$, Yi RU ${ }^{\text {a }}$, Jona THAI ${ }^{\mathrm{a}}$ \\ ${ }^{a}$ Department of Mechanical and Industrial Engineering, University of Toronto, Toronto, \\ Ontario, Canada
}

\begin{abstract}
Classical mereology is based on the assumption that any two underlapping elements have a sum, yet there are many domains (such as manufacturing assemblies, molecular structure, gene sequences, and convex time intervals) in which this assumption is not valid. In such domains, mereological sums must be connected objects. However, there has been little work in providing an axiomatization of such a mereology. Based on the observation that the underlying structures in these domains are represented by graphs, we propose a new mereotopology that axiomatizes the connected induced subgraph containment ordering for a graph, and then identify an axiomatization of the mereology that is a module of the mereotopology.
\end{abstract}

Keywords. mereology, mereotopology, lattices, graphs

\section{Introduction}

From its inception, research in mereology has been dominated by two presuppositions. One has focused on what has come to be known as classical mereology, in which underlapping elements have sums and overlapping elements have products. The other presupposition, known as mereological monism, is based on the idea that there is a single parthood relation that applies to all domains, whether they be spatial regions, temporal intervals, physical objects, or activities.

More recently, mereological pluralism has been posited in $[1,2]$, in which there are multiple distinct parthood relations for different classes of objects. Furthermore, there are a wide variety of domains (such as manufacturing assemblies, molecular structure, gene sequences, and time intervals) in which we need mereologies for connected substructures, not arbitrary substructures. The problem is that existing mereologies (such as classical extensional mereology) are too strong to represent connected substructures, that is, they allow models in which disconnected elements have mere sums. We therefore want to address the following challenge:

What is the mereology for connected substructures of a structure?

In designing an ontology, our objective is twofold - first, to prove that the models of the ontology are actually the intended models, and second, to demonstrate that the intended models do indeed formalize the ontological commitments. Our strategy is to first specify a class of mathematical structures and show that the ontology axiomatizes this class of structures (that is, there is a one-to-one correspondence between the class of models of the ontology and the class of mathematical structures). We then specify a representation theorem for this class of mathematical structures to demonstrate that it formalizes the ontological commitments. The primary benefit of this strategy is that 
it makes explicit the modular organization of the subtheories of the ontology, thereby highlighting how other ontologies are reused.

We therefore begin by presenting a series of motivating scenarios from diverse domains in which the underlying structures are graphs, and we seek the mereologies for connected induced subgraphs of these graphs. After showing that existing mereologies are inadequate for capturing these scenarios, we propose new mereotopologies that axiomatize the connected induced subgraph containment ordering for a graph. We then identify the axiomatization of the mereology that is a subtheory of the mereotopology.

\section{Motivating Scenarios}

\subsection{Assemblies and Components}

A three leg table as shown in Figure 1(i) has a topological structure as seen in Figure 1(ii). Table top $a$ is connected to all the legs $b, c$, and $d$, while all the legs are disconnected from each other. Connected components (e.g., $a$ and $b$ ) can have sums that correspond to subassemblies, while disconnected components (e.g., $b$ and $c$ ) do not constitute subassemblies, and hence do not have sums. The complete set of subassemblies for the table is shown in Figure 1(iii). Similarly, for a picture frame, the bars $a, b, c$, and $d$ topologically form a cyclic graph (see Figure 2). Bars $a, c$ and bars $b, d$ are not directly connected, so there is no sum for each of these two pairs. The mereologies for these two examples are shown in Figure 1(iv) and Figure 2(iv), and it is clear that these are not classical mereologies.

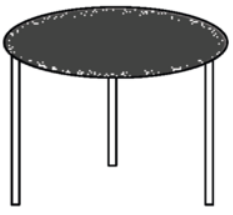

(i)

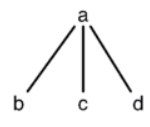

(ii)

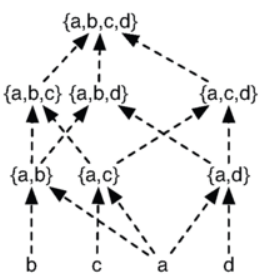

(iii)

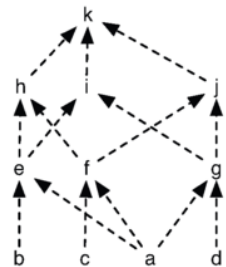

(iv)

Figure 1. Mereology on the components of a table.

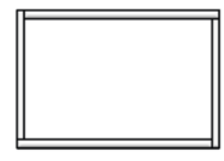

(i)

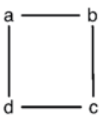

(ii)

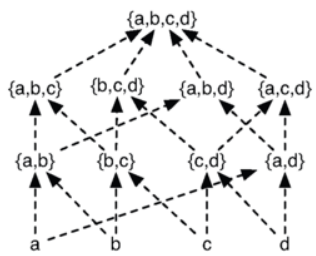

(iii)

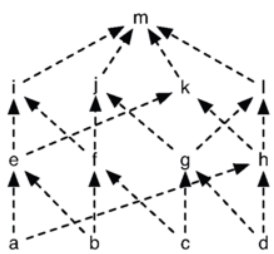

(iv)

Figure 2. Mereology on the components of a picture frame. 


\subsection{Convex Time Intervals}

Relations over temporal intervals have been foundational for qualitative temporal reasoning and representation. An early proposal for the axiomatization of an ontology of time intervals was the work of van Benthem [3], in which there is one primitive ordering relation and one primitive mereological relation over intervals. Notably, the intervals in the models of this ontology are convex - every interval between two other subintervals is also a subinterval ${ }^{1}$. In Figure 3(i), there is no interval that is the sum of intervals a and $\mathbf{c}$; although interval $\mathbf{f}$ is the least upper bound of $\mathbf{a}$ and $\mathbf{c}$, it contains the interval $\mathbf{b}$ which is disjoint from a and c. The mereology in Figure 3(ii) is not classical.

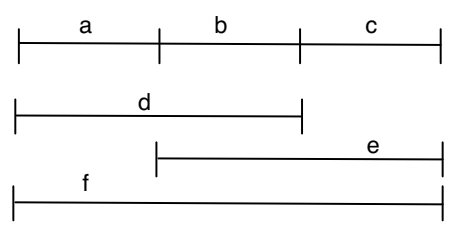

(i)

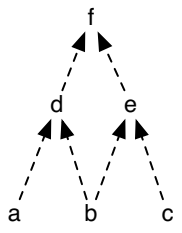

(ii)

Figure 3. Mereology on convex time intervals.

\subsection{Molecular Structure Ontology (MoSt)}

MoSt [4] combines conventional graph theory and ontological approaches to describe the shape of molecules. This ontology allows us to consider molecules from the shape perspective by identifying basic functional groups of the ring and chain types, and to use the axioms of the ontology to combine these functional groups together.

A skeleton in MoSt is the composition of one or more functional groups that are attached together. Skeletons can be composed of other skeletons - they allow us to partition the structure of molecules into various pieces, along with also combining pieces together. Because we allow various decompositions of molecules, we also must permit the notion that multiple skeletons can be formed from the combination of primitive functional groups with other groups or other atoms. We introduce a parthood relation called $\operatorname{part}(x, y)$ that outlines how two skeletons $x$ and $y$ are part of each other if and only if all elements found inside one skeleton are also found in the other skeleton:

$$
(\forall x \forall y(\operatorname{part}(x, y) \equiv(\operatorname{skeleton}(x) \wedge \operatorname{skeleton}(y) \wedge \forall z((\operatorname{mol}(z, x) \supset \operatorname{mol}(z, y))))))
$$

This is exemplified with Figure 4, where $s_{3}$ is composed of $s_{1}$ and the skeleton for $g_{3}$. We can state that " $s_{1}$ is part of $s_{3}$." We are again faced with the question: What mereology corresponds to this definition of parthood?

\footnotetext{
${ }^{1}$ van Benthem recognizes the need to axiomatize convex intervals, but he does not provide an explicit axiomatization of the mereology alone.
} 


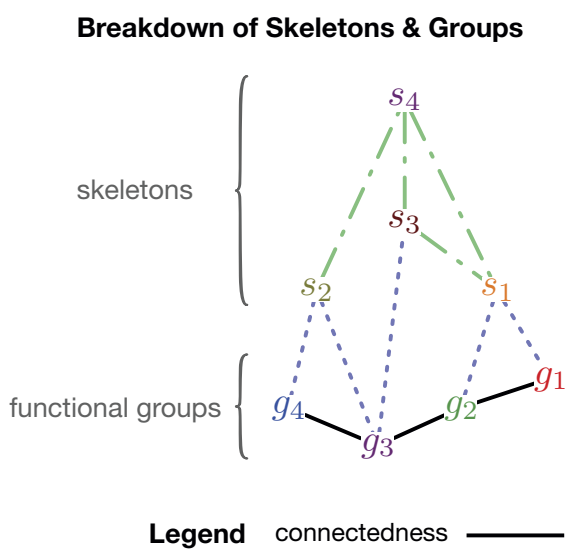

Corresponding Skeletal Diagrams
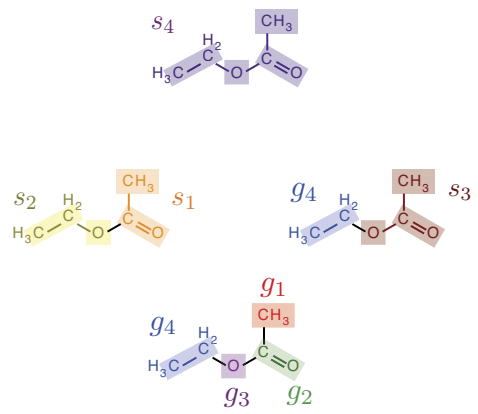

mereology - : -

Figure 4. Composition of the skeleton for ethyl acetate. $g_{1}, g_{2}, g_{3}$, and $g_{4}$ signify the primitive functional groups, and $s_{1}, s_{2}, s_{3}$, and $s_{4}$ signify the skeletons, respectively. The primitive functional groups are connected via the $\operatorname{mol}(x, y)$ relation using the dark, bolded black lines. Skeletons that contain functional groups are outlined in the dotted blue lines in the figure; for example, $s_{2}$ consists of $g_{3}$ and $g_{4}$. Green dash-dotted lines show parthood between skeletons.

\subsection{Gene Sequences}

A gene is a sequence of nucleotides that encodes the synthesis of proteins. A reading frame is a way of dividing such a sequence of nucleotides into a set of consecutive, nonoverlapping triplets (shown in Figure 5(i)). We can therefore specify a mereology on gene sequences; for example, given the sequence in Figure 5(ii), the containment ordering in Figure 5(iii) is isomorphic to the mereology in Figure 5(iv).

(i)

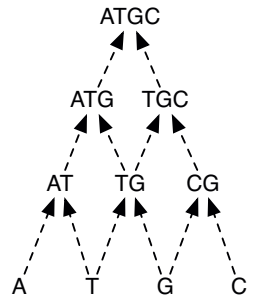

(iii)

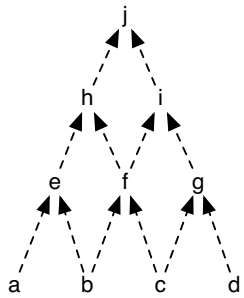

(iv)

Figure 5. Mereology on nucleotides in a gene sequence. 


\section{Relationship to Existing Mereotopologies}

Classical mereology is a formal theory of the part-whole relation [5]; in particular, it is a theory which captures how parts can be combined to form wholes and how wholes can be decomposed into parts. Pietruszczak [6] goes so far as to say that mereology arose as a theory of sums. As posited by Fine [2] and Sider [7], the prevalent intuition is that "a whole is a mere sum, or aggregate or fusion, formed from its parts without regard for how they might fit together or be structured within a more comprehensive whole." All of this is captured by $T_{c m \_m e r e o l o g y}{ }^{2}$, in which any pair of overlapping elements have a product and any pair of underlapping elements has a sum (although the precise definition of sum varies across different axiomatizations [5]).

Nevertheless, in domains such as manufacturing assembly, molecules, gene sequences, and convex time intervals, not all underlapping elements have sums. Thus, no mereology that extends $T_{\text {cm_mereology }}$ can be used to represent the scenarios in Section 2. It is clear that the mereologies we need in the motivating scenarios are not classical, since sums do not exist for all pairs of elements in the mereologies of Figure 1, 3, and 5.

Simons [8] addresses this problem in his discussion of integral wholes. He correctly notes that one commonality among all of the above scenarios is that the objects we are considering must be connected. The assembled table is distinct from the set of tabletop and legs scattered on the floor. A skeleton within a molecule must be connected - it never consists of two disconnected functional groups within a molecule. Why not leave the mereology to be classical and capture the notion of connectedness using mereotopology? In fact, Whitehead proposed a nonclassical mereology (i.e., one in which not all pairs of elements have sums) based on the notion of self-connected objects [8]. This approach has been criticized in [9] from the perspective of attempting to define connection with respect to parthood, but it was not considered as an independent mereotopological axiom.

In this paper, we are not proposing a mereology for all objects; for example, a mereology of space needs to cover all spatial regions, and not be restricted to connected regions. Instead, we are proposing a mereology that is satisfied by different specific classes of entities, such as assembled physical objects, molecules, gene sequences, and convex time intervals. Of course, there do exist objects for which sums are not connected; for example, a bikini is an object whose parts are disconnected, and the United States is a geographical entity whose parts are not connected. Indeed, this is the primary reason for adopting mereological pluralism - not all classes of objects have the same parthood relation satisfying equivalent axioms. For example, the parthood relation for convex time intervals is distinct from the parthood relation for arbitrary time intervals; the latter can be represented using a classical mereology, while the former cannot.

\section{Semantic Requirements: Connected Induced Subgraph Structures}

If we take a closer look at the motivating scenarios, we can get a sense of what requirements we need to impose on the models of the mereology that we need. We begin with the observation that each of the motivating scenarios involves subgraphs of a simple graph.

Definition 1 A graph is a pair of sets $\mathbb{G}=\langle V, \mathbf{E}\rangle$ such that

\footnotetext{
${ }^{2}$ http://colore.oor.net/mereology/cm_mereology.clif
} 
1. $\mathbf{E} \subseteq V \times V$

2. $\mathbf{E}=\mathbf{E}^{-}$;

$\mathbb{G}$ is simple iff $I \cap \mathbf{E}=\emptyset . \quad \mathbb{G}$ is a graph with loops iff $I \subseteq \mathbf{E}$.

In the case of assembled products, the underlying graph is the connection relation between non-decomposable components; subassemblies correspond to subgraphs of the graph that corresponds to the entire object. Molecules within the MoSt Ontology are specified by a graph consisting of functional groups, and skeletons correspond to subgraphs of the graph of the entire molecule. With convex time intervals and gene sequences, we have the special class of path graphs, and each sequence forms another path that is a subgraph of the entire graph.

Definition 2 Let $\mathbb{H}_{1}=\left\langle V_{1}, \mathbf{E}_{\mathbf{1}}\right\rangle$ and $\mathbb{H}_{2}=\left\langle V_{2}, \mathbf{E}_{\mathbf{2}}\right\rangle$ be simple graphs.

$\mathbb{H}_{2}$ is a subgraph of $\mathbb{H}_{1}$ (denoted by $\mathbb{H}_{2} \subseteq \mathbb{H}_{1}$ ) iff $V_{2} \subseteq V_{1}$ and $\mathbf{E}_{\mathbf{2}} \subseteq \mathbf{E}_{\mathbf{1}}$.

$\mathbb{H}_{2}$ is an induced subgraph of $\mathbb{H}_{1}$ (denoted by $\mathbb{H}_{2} \preceq \mathbb{H}_{1}$ ) iff

- $\mathbb{H}_{2}$ is a subgraph of $\mathbb{H}_{1}$;

- For any $\mathbf{x}, \mathbf{y} \in V_{2}$, if $(\mathbf{x}, \mathbf{y}) \in \mathbf{E}_{\mathbf{1}}$ then $(\mathbf{x}, \mathbf{y}) \in \mathbf{E}_{\mathbf{2}}$.

The subgraph of $\mathbb{H}_{1}$ induced by a subset $V \subseteq V_{1}$ is denoted by $\mathbb{H}_{1}[V]$.

From the motivating scenarios, we can see that we are not interested in arbitrary subgraphs of a graph $\mathbb{H}$, but rather in subgraphs that are connected (as noted in [8]). For example, the subassemblies of an assembled product are always considered to be connected. Also, each gene forms a convex interval within the entire graph that represents the genetic sequence. Within graphs, we can formalize connectedness as follows:

Definition 3 Let $\mathbb{H}=\langle V, \mathbf{E}\rangle$ be a simple graph.

$\mathbb{H}$ is a path iff $V=\mathbf{x}_{\mathbf{1}}, \ldots, \mathbf{x}_{\mathbf{n}}$ and $\mathbf{E}=\left\{\left(\mathbf{x}_{\mathbf{0}}, \mathbf{x}_{\mathbf{1}}\right), \ldots,\left(\mathbf{x}_{\mathbf{n}-\mathbf{1}}, \mathbf{x}_{\mathbf{n}}\right)\right\}$.

$\mathbb{H}$ is connected iff for any two vertices $\mathbf{x}, \mathbf{y} \in V$, there exists an induced subgraph that is a path containing $\mathbf{x}, \mathbf{y}$.

The motivating scenarios therefore lead us to focus on the set of connected induced subgraphs of the simple graph $\mathbb{H}$. It is easy to see that the induced subgraph relation is a partial ordering on the set of all connected induced subgraphs of $\mathbb{H}$, in which elements of $V$ are atoms (since they cannot have any nontrivial subgraphs). For example, in Figure 1, the induced connected subgraph corresponding to the subassembly $\{\mathbf{a}, \mathbf{c}\}$ is contained in the connected induced subgraphs $\{\mathbf{a}, \mathbf{b}, \mathbf{c}\},\{\mathbf{a}, \mathbf{c}, \mathbf{d}\}$. We can also specify a topology on the connected induced subgraphs of $\mathbb{H}$. For example, in Figure 2, the subassemblies $\{\mathbf{a}, \mathbf{b}\}$ and $\{\mathbf{c}, \mathbf{d}\}$ are disjoint as subgraphs, but are connected because $(\mathbf{b}, \mathbf{c}),(\mathbf{a}, \mathbf{d}) \in \mathbf{E}$.

Definition 4 Let $\mathbb{H}=\langle V, \mathbf{E}\rangle$ be a simple graph, and suppose

- $\mathscr{C}(\mathbb{H})=\{\mathbb{J}: \mathbb{J} \preceq \mathbb{H}, \mathbb{J}$ connected $\}$;

- $\mathscr{E}(\mathbb{H}) \subseteq \mathscr{C}(\mathbb{H}) \times \mathscr{C}(\mathbb{H})$ such that $\left(\mathbb{H}_{1}, \mathbb{H}_{2}\right) \in \mathscr{E}(\mathbb{H})$ iff $\left(V_{1} \times V_{2}\right) \cap \mathbf{E} \neq \emptyset$.

The connected induced subgraph structure of $\mathbb{H}$ is $\mathbb{C}^{\mathbb{H}}=\langle\mathscr{C}(\mathbb{H}), \preceq, \mathscr{E}(\mathbb{H})\rangle$.

We can now specify the fundamental semantic requirement for the mereology we seek: 
The models of the mereology must be representable by the following class of structures: $\mathfrak{C}=\left\{\mathbb{C}^{\mathbb{H}}: \mathbb{H} \in \mathfrak{M}^{\text {simple_graph }}\right\}$.

The next challenge is to find an axiomatization that satisfies this requirement.

\section{Using Mereotopologies for Connected Substructures}

We can use theories from the $\mathbb{H}^{\text {combined_mereotopology }}$ Hierarchy ${ }^{3}$ of the Common Logic Ontology REpository (COLORE) ${ }^{4}$ to characterize the mereologies that we are seeking. In this section, we seek a deeper understanding of the properties of the connected induced subgraph containment ordering $\mathbb{C}^{\mathbb{H}}$ for a simple graph $\mathbb{H}$. Using these properties, we define the class of intended models for the mereotopologies of connected subgraphs that can be represented by such a containment ordering. Finally, we identify a theory in the $\mathbb{H}^{\text {combined_mereotopology }}$ Hierarchy of COLORE that axiomatizes this class of structures.

\subsection{Properties of $\mathbb{C}^{\mathbb{H}}$}

The crucial insight is that the set of connected induced subgraphs of a simple graph $\mathbb{H}$ can be characterized by the relationships among the subgraphs. It is easy to see how $\mathbb{C}^{\mathbb{H}}$ can be constructed from $\mathbb{H}$ - we simply extract all connected induced subgraphs, and the containment and connection relations are already determined by their relationship to $\mathbb{H}$.

Definition 5 For each $\mathbb{K} \in \mathscr{C}(\mathbb{H})$, the set of subgraphs of the graph $\mathbb{H}$ that overlap a given subgraph is denoted by $\mathscr{O}(\mathbb{K})=\{\mathbb{J}: \mathbb{K} \cap \mathbb{J} \neq \emptyset, \mathbb{J} \in \mathscr{C}(\mathbb{H})\}$

For each $\mathbb{K} \in \mathscr{C}(\mathbb{H})$, the set of subgraphs of the graph $\mathbb{H}$ that are connected a given subgraph is denoted by $\mathscr{N}(\mathbb{K})=\left\{\mathbb{J}:(\mathbf{x}, \mathbf{y}) \in E, \mathbf{x} \in V_{J}, \mathbf{y} \in V_{K}, \mathbb{J} \in \mathscr{C}(\mathbb{H})\right\}$

The basic operation for constructing connected induced subgraphs is the following:

Definition 6 Let $\mathbb{H}_{1}=\left\langle V_{1}, \mathbf{E}_{\mathbf{1}}\right\rangle, \mathbb{H}_{2}=\left\langle V_{2}, \mathbf{E}_{\mathbf{2}}\right\rangle, \mathbb{H}_{3}=\left\langle V_{3}, \mathbf{E}_{\mathbf{3}}\right\rangle$ be connected simple graphs.

$\mathbb{H}_{1}$ is the sum of $\mathbb{H}_{2}$ and $\mathbb{H}_{2}$ (denoted by $\mathbb{H}_{1}=\mathbb{H}_{2}+\mathbb{H}_{3}$ ) iff

- $\mathbb{H}_{2} \preceq \mathbb{H}_{1}$;

- $\mathbb{H}_{3} \preceq \mathbb{H}_{1}$;

- $\mathbb{H}_{1}=\mathbb{H}_{1}\left[V_{2} \cup V_{3}\right]$.

The key to the characterization theorem is to identify the properties that the set of subgraphs must satisfy so that we can reconstruct $\mathbb{H}^{5}$.

Theorem 1 Suppose $\mathbb{H}$ is a simple graph.

Let $\mathscr{C}$ be a set of connected induced subgraphs of $\mathbb{H}$, and suppose $\mathscr{E} \subseteq \mathscr{C} \times \mathscr{C}$ such that $\left(\mathbb{H}_{1}, \mathbb{H}_{2}\right) \in \mathscr{E}$ iff $\left(V_{1} \times V_{2}\right) \cap \mathbf{E} \neq \emptyset$.

$\langle\mathscr{C}, \preceq, \mathscr{E}\rangle$ is the connected induced subgraph structure of $\mathbb{H}$ iff the following conditions are satisfied:

\footnotetext{
${ }^{3}$ http://colore.oor.net/combined_mereotopology

${ }^{4}$ http://colore.oor.net/

${ }^{5}$ The full version of this paper, containing proofs for all results, can be found at http://stl.mie. utoronto.ca/publications/full_cisco.pdf
} 
1. Any two connected induced subgraphs of $\mathbb{H}$ are contained in another connected induced subgraph: If $\mathbb{H}_{1}, \mathbb{H}_{2} \in \mathscr{C}$, then there exists $\mathbb{H}_{3} \in \mathscr{C}$ such that

$$
\mathbb{H}_{1} \preceq \mathbb{H}_{3}, \mathbb{H}_{2} \preceq \mathbb{H}_{3}
$$

2. The containment ordering $\preceq$ is preserved by the combination of a graph and its subgraphs: If $\mathbb{H}_{1}, \mathbb{H}_{2} \in \mathscr{C}$, then

$$
\mathbb{H}_{1} \preceq \mathbb{H}_{2} \Leftrightarrow \mathbb{H}_{2}=\mathbb{H}_{1}+\mathbb{H}_{2}
$$

3. For any two subgraphs of $\mathbb{H}$ that are connected to each other, there exists a subgraph of $\mathbb{H}$ that is their sum: $\left(\mathbb{H}_{1}, \mathbb{H}_{2}\right) \in \mathscr{E}$ iff there exists $\mathbb{H}_{3} \in \mathscr{E}$ such that

$$
\mathbb{H}_{3}=\mathbb{H}_{1}+\mathbb{H}_{2}
$$

4. The composition of two subgraphs is equal to the mereological and topological sums of the subgraphs: If $\mathbb{H}_{1}, \mathbb{H}_{2}, \mathbb{H}_{3} \in \mathscr{C}$, then

$$
\begin{gathered}
\mathbb{H}_{3}=\mathbb{H}_{1}+\mathbb{H}_{2} \Leftrightarrow \mathscr{O}\left(\mathbb{H}_{3}\right)=\mathscr{O}\left(\mathbb{H}_{1}\right) \cup \mathscr{O}\left(\mathbb{H}_{2}\right) \\
\mathbb{H}_{3}=\mathbb{H}_{1}+\mathbb{H}_{2} \Rightarrow \mathscr{N}\left(\mathbb{H}_{3}\right)=\mathscr{N}\left(\mathbb{H}_{1}\right) \cup \mathscr{N}\left(\mathbb{H}_{2}\right)
\end{gathered}
$$

5. Every connected induced subgraph of $\mathbb{H}$ can be decomposed into the sum of a trivial subgraph and a connected induced subgraph: If $\mathbb{H}_{1} \in \mathscr{C}$, then there exists $\mathbb{H}_{2}, \mathbb{H}_{3} \in \mathscr{C}$ such that $\mathbb{H}_{2} \cong K_{1}$ and

$$
\mathbb{H}_{1}=\mathbb{H}_{2}+\mathbb{H}_{3}
$$

A careful inspection of the structures in Figures 1(iii), 2(iii), 3(ii), and 5(iii) reveals that each of them do indeed satisfy the conditions in Theorem 1, so that they are the connected induced subgraph structures for their respective graphs. In other words, we have the right set of structures to use as the basis for a representation theorem.

\subsection{Mereographs for Connected Induced Subgraphs}

What is the mereotopology that axiomatizes the connected induced subgraph structure for a simple graph $\mathbb{H}$ ?

\subsubsection{Mereographs and the Mereotopology $M T$}

We follow the work of [10] for the approach to mereotopology in which both parthood and connection are primitive relations. The mereology of the parthood relation is represented by the class of partial orderings $\mathfrak{M}^{\text {partial_ordering }}$, and the connection relation is represented by the class of graphs with loops $\mathfrak{M}^{\text {graph_loops }}$.

Definition 7 Suppose $\mathbb{P} \in \mathfrak{M}^{\text {partial_ordering }}$ such that $\mathbb{P}=\langle V, \preceq\rangle$.

The upper set for $\mathbf{x}$ in $\mathbb{P}$, denoted by $U^{\mathbb{P}}(\mathbf{x})$, is

$$
U^{\mathbb{P}}(\mathbf{x})=\{\mathbf{y}: \mathbf{x} \leq \mathbf{y}\} \quad U^{\mathbb{P}}(X)=\bigcup_{\mathbf{x} \in X} U(\mathbf{x})
$$

$\mathscr{L}_{\mathbb{P}}=\langle V, E\rangle$ is the lower bound graph for $\mathbb{P}:(\mathbf{x}, \mathbf{y}) \in E \quad L^{\mathbb{P}}[\mathbf{x}] \cap L^{\mathbb{P}}[\mathbf{y}] \neq \emptyset$ 
Definition 8 Suppose $\mathbb{G} \in \mathfrak{M}^{\text {graph_loops }}$, such that $\mathbb{G}=\langle V, \mathbf{E}\rangle$.

The neighbourhood of $\mathbf{x}$ in $\mathbb{G}$, denoted by $N^{\mathbb{G}}(\mathbf{x})$, is

$$
N^{\mathbb{G}}(\mathbf{x})=\{\mathbf{y}:(\mathbf{x}, \mathbf{y}) \in \mathbf{E}\} \quad N^{\mathbb{G}}(X)=\bigcup_{\mathbf{x} \in X} N^{\mathbb{G}}(\mathbf{x})
$$

A new class of mathematical structures was introduced by [10] to characterize the models of mereotopological theories.

Definition $9 \mathbb{P} \oplus \mathbb{G}=\langle V, \mathbf{E}, \leq\rangle$ is a mereograph iff

1. $\mathbb{P}=\langle V, \leq\rangle$ such that $\mathbb{P} \in \mathfrak{M}^{\text {partial_ordering }}$;

2. $\mathbb{G}=\langle V, \mathbf{E}\rangle$ such that $\mathbb{G} \in \mathfrak{M}^{\text {graph_loops }}$;

3. $U^{\mathbb{P}}\left(N^{\mathbb{G}}(\mathbf{x})\right) \subseteq N^{\mathbb{G}}(\mathbf{x})$, for each $\mathbf{x} \in V$.

$\mathfrak{M}^{\text {mereograph }}$ denotes the class of mereographs.

In other words, a mereograph is the amalgamation of partial orderings and graphs with loops, where Condition (3) constrains how these two structures are related to each other: the neighbourhood of a vertex in the graph is closed under upper sets in the partial ordering. Consequently, mereographs are the right class of structures that we need:

Lemma 1 If $\mathbb{H}$ is a simple graph, then $\mathbb{C}^{\mathbb{H}}=\langle\mathscr{C}(\mathbb{H}), \preceq, \mathscr{E}\rangle$ is a mereograph.

The next question is to determine exactly what class of mereographs we need.

\subsubsection{Connected Induced Subgraph Mereographs}

Our goal is to specify the conditions that a mereograph must satisfy if it is to be representable by $\mathbb{C}^{\mathbb{H}}$. The approach we take is to "translate" the properties of $\mathbb{C}^{\mathbb{H}}$ (proven in Theorem 1) into properties of mereographs.

Definition 10 Suppose

$\Sigma(\mathbf{x}, \mathbf{y})=\left\{\mathbf{z}: N^{\mathscr{L}(\mathbb{P})}[\mathbf{z}]=N^{\mathscr{L}(\mathbb{P})}[\mathbf{x}] \cup N^{\mathscr{L}(\mathbb{P})}[\mathbf{y}]\right\}$

$\Sigma^{-1}(\mathbf{x})=\{(\mathbf{y}, \mathbf{z}): \Sigma(\mathbf{y}, \mathbf{z})=\mathbf{x}\}$

$\Pi(\mathbf{x}, \mathbf{y})=\left\{\mathbf{z}: N^{\mathbb{G}}[\mathbf{z}]=N^{\mathbb{G}}[\mathbf{x}] \cup N^{\mathbb{G}}[\mathbf{y}]\right\}$

A mereograph $\mathbb{P} \oplus \mathbb{G}=\langle V, \mathbf{E}, \leq\rangle$ is a connected induced subgraph mereograph iff

1. $U^{\mathbb{P}}[\mathbf{x}] \cap U^{\mathbb{P}}[\mathbf{y}] \neq \emptyset$;

2. $U^{\mathbb{P}}[\mathbf{x}] \subseteq U^{\mathbb{P}}[\mathbf{y}]$ iff $\mathscr{L}^{\mathbb{P}}(\mathbf{x}) \subseteq \mathscr{L}^{\mathbb{P}}(\mathbf{y})$;

3. $\mathbf{y} \in N^{\mathbb{G}}[\mathbf{x}]$ iff $\Sigma(\mathbf{x}, \mathbf{y}) \neq \emptyset$ for any $\mathbf{x}, \mathbf{y} \in V$;

4. $\Sigma(\mathbf{x}, \mathbf{y}) \subseteq \Pi(\mathbf{x}, \mathbf{y})$, for any $\mathbf{x}, \mathbf{y} \in V$;

5. $\Sigma^{-1}(\mathbf{x}) \subseteq(\mathscr{A}(\mathbb{P}) \times V)$, where $\mathscr{A}(\mathbb{P})$ is the set of atoms in $\mathbb{P}$.

$\mathfrak{M}^{\text {cisco } m t}$ denotes the class of connected induced subgraph mereographs ${ }^{6}$.

\footnotetext{
${ }^{6}$ The name "cisco" is an acronym for "connected induced subgraph containment ordering."
} 


\subsubsection{Representation Theorems for Connected Induced Subgraphs}

Now that we have defined the class of connected induced subgraph mereographs, we can demonstrate that they are the correct set of structures by proving that they are indeed representable by the connected induced subgraph structures of simple graphs.

Theorem 2 There is a bijection $\varphi: \mathfrak{M}^{\text {ciscomt }} \rightarrow \mathfrak{C}$ and an isomorphism

$$
\mu: \mathbb{P} \oplus \mathbb{G} \rightarrow \varphi(\mathbb{P} \oplus \mathbb{G})
$$

This constitutes the representation theorem for connected induced subgraph mereographs. As such, it also provides a validation of this class of mereographs as the right class of structures to be the intended models of the axiomatization of the mereotopology, which we now consider.

\subsection{Connected Sums of Subgraphs}

The next step is to provide an axiomatization of the class of connected induced subgraph mereographs. Before moving to the axiomatization of the mereotopology for connected induced subgraphs, we briefly prove some properties of sums and the relationship to the theory $T_{\text {em mereology }}$ (Strong Supplementation) that we will need later in the paper.

\subsubsection{Strong Supplementation and Properties of Sums}

Classical mereology $T_{\text {cmımereology }}{ }^{7}$ entails the existence of the sum of any two underlapping elements. Although we are seeking a logical theory that is weaker than $T_{\text {cm mereology }}$, we still need to adopt a definition for mereological sum. In classical mereology, there are actually different axiomatizations for the mereological sum of two elements [11], but we adopt the following:

Definition $11 T_{\text {sumdef }}$ is the definitional extension of $T_{m \_m e r e o l o g y}$ with the sentence ${ }^{8}$ :

$$
(\forall x \forall y \forall z(\operatorname{sum}(x, y, z) \equiv(\forall u(\operatorname{overlaps}(u, z) \equiv(\operatorname{overlaps}(u, x) \vee \operatorname{overlaps}(u, y))))))
$$

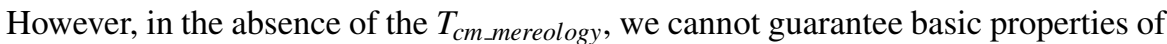
sums, such as functionality or its relationship to parthood. In fact, there is a close relationship between the Strong Supplementation Principle (axiomatized by $T_{\text {em_mereology }}{ }^{9}$ ), the required properties of mereological sums, and weaker supplementation principles.

Proposition 1 Let $T_{\text {sumpart }}$ be the extension of $T_{\text {sumdef }}$ with the sentence ${ }^{10}$ :

$$
(\forall x \forall y \forall z(\operatorname{sum}(x, y, z) \supset \operatorname{part}(x, z)))
$$

$T_{\text {em_mereology }}$ is logically equivalent to $T_{m m \_m e r e o l o g y}{ }^{11} \cup T_{\text {sumpart }}$.

Proof: http://colore.oor.net/mereology/theorems/sumpart/

\footnotetext{
${ }^{7}$ http://colore.oor.net/mereology/cm_mereology.clif

${ }^{8}$ http://colore.oor.net/mereology/definitions/sum.clif

${ }^{9}$ http://colore.oor.net/mereology/em_mereology.clif

${ }^{10}$ http://colore.oor.net/mereology/sumpart.clif

${ }^{11}$ Weak Supplementation Principle: http://colore.oor.net/mereology/mm_mereology.clif
} 
Proposition 2 Let $T_{\text {sumfun }}$ be the extension of $T_{\text {sumdef }}$ with the sentence ${ }^{12}$ :

$$
(\forall x \forall y \forall z \forall u(\operatorname{sum}(x, y, z) \wedge \operatorname{sum}(x, y, u) \supset(z=u)))
$$

$T_{\text {em_mereology }}$ is logically equivalent to $T_{\text {ppp_mm_mereology }}{ }^{13} \cup T_{\text {sumfun }}$.

Proof: http://colore.oor.net/mereology/theorems/sumfun/

Finally, we can show that the Strong Supplementation Principles is equivalent to the relationship between parthood and sums which corresponds to Condition (2) in Theorem 1:

Proposition $3 T_{\text {em_mereology }}$ is logically equivalent ${ }^{14}$ to the extension of $T_{\text {sumdef }}$ by:

$$
(\forall x \forall y(p a r t(x, y) \equiv \operatorname{sum}(x, y, y)))
$$

Proof: http://colore.oor.net/mereology/theorems/emsum/

This is interesting in the light of Fine's notion of operationalism, in which the parthood relation is defined in terms of composition operations like sums, rather than defining sums in terms of the parthood relation.

\subsubsection{Mereotopology of Connected Sums}

The mereotopology we have been pursuing can be obtained by a rather straightforward axiomatization $^{15}$ of the conditions in the definition of $\mathfrak{M}^{\text {cisco_mt }}$.

Definition $12 T_{\text {cisco } m t}$ is the extension ${ }^{16}$ of $T_{m t} \cup T_{\text {em_mereology }} \cup T_{\text {ub_mereology }} \cup T_{\text {sumdef }}$

$$
\begin{aligned}
& (\forall x \forall y(C(x, y) \equiv(\exists z(\operatorname{sum}(x, y, z))))) \\
& (\forall x \forall y \forall z(\operatorname{sum}(x, y, z) \supset(\forall u(C(u, z) \equiv(C(u, x) \vee C(u, y)))))) \\
& (\forall x \exists y \exists z(\operatorname{atom}(y) \wedge \operatorname{sum}(y, z, x)))
\end{aligned}
$$

$T_{u b \_m e r e o l o g y}$ corresponds to Condition (1) in Definition 10, and by Proposition 3, $T_{\text {em_mereology }}$ corresponds to Condition (2) in Definition 10. The remaining axioms in $T_{\text {cisco_mt }}$ correspond to Conditions (3) to (5), respectively, in Definition 10.

Formalizing these correspondences gives us the following result, which is the verification of $T_{\text {cisco_mt }}$, and shows that we have the correct set of axioms:

Theorem 3 There exists a bijection $\varphi: \operatorname{Mod}\left(T_{\text {cisco_mt }}\right) \rightarrow \mathfrak{M}^{\text {cisco_mt }}$ such that

1. $\langle\mathbf{x}, \mathbf{y}\rangle \in \mathbf{C}^{\mathscr{M}} \quad$ iff $\quad \mathbf{y} \in N^{\mathbb{G}}[\mathbf{x}]$;

2. $\langle\mathbf{x}, \mathbf{y}\rangle \in \operatorname{part}^{\mathscr{M}} \quad$ iff $\quad \mathbf{x} \in L^{\mathbb{P}}[\mathbf{y}]$.

3. $\langle\mathbf{x}, \mathbf{y}, \mathbf{z}\rangle \in \mathbf{s u m}^{\mathscr{M}} \quad$ iff $\Sigma(\mathbf{x}, \mathbf{y})=\{\mathbf{z}\}$;

\footnotetext{
${ }^{12}$ http://colore.oor.net/mereology/sumfun.clif

${ }^{13}$ Proper Parts Principle: http://colore.oor.net/mereology/ppp_mm_mereology.clif

${ }^{14}$ In [11], Varzi shows that $T_{\text {em_mereology }}$ entails the sentence in Proposition 3, but does not establish the equivalence.

${ }^{15}$ Axiom (5) is equivalent to Simons' [8] combination of axiom (TID8) from Tiles and (WD5) from Whitehead.

${ }^{16}$ http://colore.oor.net/combined_mereotopology/cisco_mt.clif
} 


\section{Weak Mereotopology of Connected Substructures}

If we revisit the motivating scenario for molecular structure, then it appears that $T_{\text {cisco } m t}$ is too strong. In Figure 4, the functional groups $\mathbf{g}_{\mathbf{2}}, \mathbf{g}_{3}$ are attached, yet there is no skeleton that is the sum of these groups, and hence we do not have a connected induced subgraph mereograph. On the other hand, the remaining conditions in Definition 10 are satisfied. We therefore consider a generalization of the class of a connected induced subgraph mereographs that can be used to capture mereotopologies such as the one in Figure 4. In particular, Condition (3) in Definition 10 is weakened to allow the existence of connected elements that do not have a sum:

Definition 13 A mereograph $\mathbb{P} \oplus \mathbb{G}=\langle V, \mathbf{E}, \leq\rangle$ is a self-connected induced subgraph mereograph iff

1. $U^{\mathbb{P}}[\mathbf{x}] \cap U^{\mathbb{P}}[\mathbf{y}] \neq \emptyset$;

2. $U^{\mathbb{P}}[\mathbf{x}] \subseteq U^{\mathbb{P}}[\mathbf{y}]$ iff $\mathscr{L}^{\mathbb{P}}(\mathbf{x}) \subseteq \mathscr{L}^{\mathbb{P}}(\mathbf{y})$;

3. $\Sigma(\mathbf{x}, \mathbf{y}) \neq \emptyset$ implies $\mathbf{y} \in N^{\mathbb{G}}[\mathbf{x}]$, for any $\mathbf{x}, \mathbf{y} \in V$;

4. $\Sigma(\mathbf{x}, \mathbf{y}) \subseteq \Pi(\mathbf{x}, \mathbf{y})$, for any $\mathbf{x}, \mathbf{y} \in V$;

5. $\Sigma^{-1}(\mathbf{x}) \subseteq(\mathscr{A}(\mathbb{P}) \times V)$.

$\mathfrak{M}^{\text {weak_cisco } m t}$ denotes the class of self-connected induced subgraph mereographs.

Since this is a generalization of the class of connected induced subgraph mereographs, we can also obtain a generalization of the representation theorem:

Theorem 4 There is a bijection $\varphi: \mathfrak{M}^{\text {weak_cisco } m t} \rightarrow \mathfrak{C}$ and a monomorphism

that fixes $\mathscr{A}(\mathbb{P})$.

$$
\mu: \mathbb{P} \oplus \mathbb{G} \rightarrow \varphi(\mathbb{P} \oplus \mathbb{G})
$$

The key difference between Theorem 2 and Theorem 4 is that, because selfconnected induced subgraph mereographs allow connected elements that do not have sums, they need not be isomorphic to a connected induced subgraph structure $\mathbb{C}^{\mathbb{H}}$. However, there still needs to be a one-to-one correspondence between the vertices of the graph $\mathbb{H}$ and the atoms of the partial ordering in the mereograph. Recalling the structure in Figure 4, we can see that it is monomorphic to the connected induced subgraph structure for the path graph $P_{4}$.

The axiomatization of the mereotopology that we need for motivating scenarios such as molecular structure in MoSt therefore only requires that sums are connected. The following result shows that this set of axioms does indeed provide the correct axiomatization of the class of self-connected induced subgraph mereographs:

Theorem 5 Let $T_{\text {weak_cisco_mt }}$ be the extension of $T_{m t} \cup T_{\text {em_mereology }} \cup T_{\text {ub_mereology }} \cup T_{\text {sumdef }}$ with the following sentences ${ }^{17}$ :

$$
\begin{gathered}
(\forall x \forall y \forall z(\operatorname{sum}(x, y, z) \supset(\forall u(C(u, z) \equiv(C(u, x) \vee C(u, y)))))) \\
(\forall x \exists y \exists z(\operatorname{atom}(y) \wedge \operatorname{sum}(y, z, x))) \\
(\forall x \forall y(\operatorname{sum}(x, y, z) \supset C(x, y)))
\end{gathered}
$$

\footnotetext{
${ }^{17}$ http://colore.oor.net/combined_mereotopology/weak_cisco_mt.clif
} 
There exists a bijection $\varphi: \operatorname{Mod}\left(T_{\text {weak_cisco_mt }}\right) \rightarrow \mathfrak{M}^{\text {weak_cisco_mt }}$ such that

1. $(\mathbf{x}, \mathbf{y}) \in \mathbf{C}^{\mathscr{M}} \quad$ iff $\quad \mathbf{y} \in N^{\mathbb{G}}[\mathbf{x}]$;

2. $(\mathbf{x}, \mathbf{y}) \in \operatorname{part}^{\mathscr{M}} \quad$ iff $\quad \mathbf{x} \in L^{\mathbb{P}}[\mathbf{y}]$.

$T_{\text {weak_cisco_mt }}$ is closely related to the notion of self-connected objects:

Proposition 4 If $T_{\text {scdef }}$ is the extension of $T_{\text {emt }} \cup T_{\text {sumdef }}$ with

$$
(\forall x(S C(x) \equiv(\forall y \forall z(\operatorname{sum}(y, z, x) \supset C(y, z)))))
$$

then $T_{\text {weak_cisco_mt }} \cup T_{\text {scdef }} \models(\forall x) S C(x)$.

\section{Mereology of Connected Subgraphs of a Graph}

Up to this point, we have characterized the mereotopology that corresponds to the connected induced subgraph containment ordering for a graph. However, the axiomatization in $T_{\text {cisco_mt }}$ uses the combined signature of mereology and topology. In this section, we identify an axiomatization of the connected induced subgraph containment ordering using only the signature of mereology, and show that such an axiomatization forms a module of $T_{\text {cisco } m t}$. This allows us to speak of the mereology of connected subgraphs of a graph.

\subsection{Subgraph Containment Lattices}

Remarkably, the problem of characterizing the class of posets which are isomorphic to the connected induced subgraph containment ordering of a graph has been posed and solved within the mathematics community [12-15]. If we re-examine the mereologies for the motivating scenarios, we see that they satisfy the following definition:

Definition 14 A partial ordering $\mathbb{P}=\langle V, \leq\rangle$ is properly semimodular iff

1. $\mathbb{P}$ is atom-height, that is, the cardinality of all maximal chains in $\mathbb{P}$ is equal to the cardinality of the set of atoms in $\mathbb{P}$;

2. for each $\mathbf{x} \in V,\left\langle U^{\mathbb{P}}[\mathbf{x}], \leq\right\rangle$ is an upper semimodular lattice:

(a) any two elements $\mathbf{y}, \mathbf{z}$ have a least upper bound and a greatest lower bound in $U^{\mathbb{P}}[\mathbf{x}]$;

(b) if $\mathbf{z}$ covers the greatest lower bound of $\mathbf{z}$ and $\mathbf{y}$, then the least upper bound of $\mathbf{z}$ and $\mathbf{y}$ covers $\mathbf{y}$.

$\mathfrak{M}^{\text {proper_semimodular }}$ denotes the class of properly semimodular partial orderings.

The central theorem shows that the class of properly semimodular partial orderings is equivalent to the connected induced subgraph ordering for a simple graph $\mathbb{H}$ :

Theorem 6 Let $\mathbb{H}=\langle V, \mathbf{E}\rangle$ be a simple graph, and let $\mathbb{P}=\langle V, \leq\rangle$ be a partial ordering. $\mathbb{P} \cong\langle\mathscr{C}(\mathbb{H}), \preceq\rangle \quad$ iff $\quad \mathbb{P} \in \mathfrak{M}^{\text {proper_semimodular }}$

Moreover, this suggests that we can axiomatize the class of connected induced subgraph orderings by axiomatizing the class of properly semimodular partial orderings. 


\subsection{Introducing $T_{\text {cisco }}$}

Using Theorem 6, we can specify a logical theory within the $\mathbb{H}^{\text {mereology }}$ Hierarchy of COLORE that is logically synonymous with the class of properly upper semimodular partial orderings.

Theorem 7 Let $T_{\text {cisco }}$ be the extension of $T_{\text {em_mereology }}$ with the sentences ${ }^{18}$ :

$$
\begin{aligned}
& (\forall u \forall x(\operatorname{ppart}(u, x) \supset(\exists y(\operatorname{atom}(y) \wedge \operatorname{part}(y, x))))) \\
& (\forall x \forall y(\operatorname{covers}(x, y) \supset(\exists z(\operatorname{atom}(z) \wedge \operatorname{ppart}(z, x) \wedge \neg \operatorname{part}(z, y))))) \\
& (\forall x \forall y \forall z \forall u((\operatorname{covers}(x, y) \wedge \operatorname{atom}(z) \wedge \operatorname{ppart}(z, x) \wedge \neg \operatorname{part}(z, y) \\
& \wedge \operatorname{atom}(u) \wedge \operatorname{ppart}(u, x) \wedge \neg \operatorname{part}(u, y)) \supset(z=u))) \\
& (\forall x \forall a \forall b((\operatorname{part}(x, a) \wedge \operatorname{part}(x, b)) \\
& \supset(\exists z(\operatorname{part}(x, z) \wedge(\forall u(\operatorname{part}(z, u) \equiv(\operatorname{part}(a, u) \wedge \operatorname{part}(b, u))))))) \\
& (\forall x \forall a \forall b((\operatorname{part}(x, a) \wedge \operatorname{part}(x, b)) \\
& \supset(\exists z(\operatorname{part}(x, z) \wedge(\forall u((\operatorname{part}(u, z) \equiv(\operatorname{part}(u, a) \wedge \operatorname{part}(u, b))))))))) \\
& (\forall p \forall x \forall y((\operatorname{atom}(p) \wedge \operatorname{part}(x, y) \wedge \neg \operatorname{part}(p, y)) \\
& \supset(\exists z(\operatorname{part}(x, z) \wedge \operatorname{part}(p, z) \wedge \operatorname{part}(y, z) \wedge \operatorname{covers}(z, y)))))
\end{aligned}
$$

There exists a bijection $\varphi: \operatorname{Mod}\left(T_{\text {cisco }}\right) \rightarrow \mathfrak{M}^{\text {proper_semimodular }}$ such that

$$
(\mathbf{x}, \mathbf{y}) \in \text { part }^{\mathscr{M}} \quad \text { iff } \quad \mathbf{x} \in L^{\mathbb{P}}[\mathbf{y}]
$$

Axioms 12, 13, and 14 guarantee that the mereology is atom-height (condition (1) of Definition 14). Axioms 15, 16, and 17 guarantee that the upper set of each element in the mereology is an upper semimodular lattice (Condition (2) of Definition 14).

Theorem 8 For any $\mathbb{P} \in \mathfrak{M}^{\text {cisco }}$ there exists a unique $\mathbb{G} \in \mathfrak{M}^{\text {graphloops }}$ such that $\mathbb{P} \oplus \mathbb{G} \in \mathfrak{M}^{\text {cisco } \_t}$

This result shows that $T_{\text {cisco } m t}$ is a conservative extension of $T_{\text {cisco }}$. Consequently, $T_{\text {cisco }}$ is indeed the mereology we seek - a new nonclassical mereology that applies to the classes of objects seen in the motivating scenarios of Section 2 .

\section{Summary}

We began this paper with the observation that classical mereology is not appropriate for certain classes of objects, such as assemblies, convex time intervals, molecules, gene sequences, because sums do not exist for every pair of such elements. This launched the quest for exactly what mereology corresponds to the parthood relation for such objects.

\footnotetext{
${ }^{18}$ http://colore.oor.net/mereology/cisco.clif
} 
A key insight is that the underlying structure that specifies an object in each of the motivating scenarios is a graph, and all parts of the object correspond to connected induced subgraphs of that graph. We therefore introduced the parthood and connection structure on the set of connected induced subgraphs of a graph and used this as the basis of the representation theorem for a new mereotopology, $T_{\text {cisco mt }}$. Finally, we specified the axiomatization of the nonclassical mereology $T_{\text {cisco }}$, which is the mereology that is conservatively extended by $T_{\text {cisco }\lrcorner t}$.

In the mereotopology $T_{\text {cisco_mt }}$, the sum of two elements exists iff they are connected. We also introduced a weaker mereotopology $T_{\text {weak_ciscomt }}$ in which not all connected elements have sums, although elements for which sums do exist must be connected. The characterization of the mereology that is the module of $T_{\text {weak_cisco_mt }}$ remains an open question.

An additional area for future work is to explore the extensions of $T_{\text {cisco }}$ that correspond to special classes of graphs. For example, in the cases of convex time intervals and gene sequences, the underlying graph is a path graph, and the resulting mereology corresponds to a special class of lattices.

\section{References}

[1] Ru Y, Grüninger M. Parts Unknown: Mereologies for Solid Physical Objects. In: Proceedings of the Joint Ontology Workshops 2017. vol. 2050. CEUR-WS.org; 2017. p. 1-9.

[2] Fine K. Towards a Theory of Part. Journal of Philosophy. 2010;107:559-589.

[3] van Benthem J. Logic of Time. Springer Verlag; 1983.

[4] Chui C, Grüninger M. A Molecular Structure Ontology for Medicinal Chemistry. In: Ferrario R, Kuhn W, editors. Formal Ontology in Information Systems - Proceedings of the 9th International Conference, FOIS 2016, Annecy, France, July 6-9, 2016. IOS Press; 2016. p. 285-298.

[5] Hovda P. What is Classical Mereology? J Philos Logic. 2009;38:55-82.

[6] Pietruszczak A. A General Concept of Being a Part of a Whole. Notre Dame Journal of Formal Logic. 2014;55:359-381.

[7] Sider T. Nothing over and above. Grazer Philosophische Studien. 1989;91:191-216.

[8] Simons P. Parts: A Study in Ontology. Oxford University Press, USA; 1987.

[9] Casati R, Varzi AC. Parts and Places: The Structures of Spatial Representation. MIT CogNet; 1999.

[10] Grüninger M, Aameri B. A New Perspective on the Mereotopology of RCC8. In: Proceeding of the 13th International Conference on Spatial Information Theory (COSIT 2017). vol. 86. Schloss Dagstuhl - Leibniz-Zentrum für Informatik; 2017. p. 2:1-2:13.

[11] Varzi C. Formal Theories of Parthood. In: Calosi C, Graziani P, editors. Mereology and the Sciences: Parts and Wholes in the Contemporary Scientific Context; 2014. p. 359-378.

[12] Niemenen J. The Lattice of Connected Subgraphs of a Connected Graph. Annales Societatis Mathematicae Polonae. 1979;21(1):187-193.

[13] Klavžar S, Petkovšek M, Steelman JH. E3281: The Lattice of Connected Subgraphs of a Graph. The American Mathematical Monthly. 1990;97(10):924-925.

[14] Kézdy A, Seif S. When is a Poset Isomorphic to the Poset of Connected Induced Subgraphs of a Graph? Southwest J Pure Appl Math. 1996;1:42-50.

[15] Smith J. The Poset of Graphs Ordered by Induced Containment. J Comb Theory Ser A. 2019;168:348373. 